\title{
Analytical Approach for Description of Ion Motion in Quadrupole Mass Spectrometer
}

\author{
Vladimir I. Baranov \\ MDS SCIEX, Concord, Ontario, Canada
}

\begin{abstract}
Implementation of the analytical method of the solution of the Mathieu equation in conjunction with the algebraic presentation of Mathieu functions is discussed in this work. This approach is used for the analytical expression of fundamental properties of the quadrupole field such as ion trajectory stability and transmission. Extensive comparison with the matrix method is presented with demonstration of the fundamental advantages of the analytical method. However, contrary to the matrix method, the analytical method is limited to the cos trapping waveforms. (J Am Soc Mass Spectrom 2003, 14, 818-824) ( 2003 American Society for Mass Spectrometry
\end{abstract}

$\mathrm{T}$ The recent development of algebraic methods to compute Mathieu functions [1-3] has a potential to simplify significantly the theoretical description of the motion of ions in a quadrupole trap. As a result of this progress the algebraic aspects of Mathieu functions were implemented in computer algebra systems such as Mathematica [4] covering a broad range of $a$ and $q$ parameters. In addition to the obvious convenience of the analytical method of solution of the Mathieu equation in conjunction with the algebraic presentation of Mathieu functions, this approach allows a single solution to be used on a complete ion trajectory. The closed formulae obtained provide an alternative method to the numerical solution of the Mathieu equation and to the matrix method. This approach also offers some possibilities for analytical expression of fundamental properties of the quadrupole field such as ion trajectory stability, transmission, and momentum/energy characteristics of ion motion.

Despite its universal power, the numerical solution of the Mathieu equation has its inherited limitations: It is difficult to distinguish between instability of a particular ion trajectory and mismatch between the ion source emittance and the quadrupole acceptance; accumulation of the computational error limits the ion residence time; the numerical solution offers limited insight even in the most powerful Monte-Carlo form. The stability of the ions' motion and their acceptance in an ideal quadrupole field with periodic time varying potentials was intensively investigated by the matrix method (for example [5, 6]). It is not limited to cos trapping waveforms and can be used directly for solution of the more general Hill equation. The matrix method requires a numerical method to calculate an ion

Published online June 25, 2003

Address reprint requests to Dr. V. I. Baranov, MDS SCIEX, 71 Four Valley Drive, Concord, Ontario L4K 4V8, Canada. E-mail: vladimir.baranov@ sciex.com trajectory over only one cycle. The major limitation of this method is associated with the absence of an analytical expression for the ion trajectory and, as a result, the necessity to involve the numerical method for averaging of the ion beam properties in the presence of the trapping potentials and collisions. The matrix method is used in the present work for direct comparison. The analytical method allows the analytical formulation of an ion trajectory over an unlimited number of cycles and does not need the matrix method. However, many achievements of the matrix method could be incorporated. Here, simple expressions for the stability conditions, acceptances, and resonances are presented. As a weakness of the analytical method of solution of the Mathieu equation in conjunction with the algebraic presentation of Mathieu functions it should be noted that the method is still limited to the cos trapping waveforms.

\section{Equations of Ion Motion}

Solutions to the Mathieu differential equation

$$
\frac{d^{2} u}{d \xi^{2}}+\left(\alpha-2 q \cos \left(2\left(\xi+\xi_{0}\right)\right)\right) u=0
$$

can be presented in terms of the Mathieu functions as follows:

$$
\begin{aligned}
& u(\xi)=K_{1} C\left(a, q, \xi+\xi_{0}\right)+K_{2} S\left(a, q, \xi+\xi_{0}\right) \\
& \dot{u}(\xi)=K_{1} \dot{C}\left(a, q, \xi+\xi_{0}\right)+K_{2} \dot{S}\left(a, q, \xi+\xi_{0}\right)
\end{aligned},
$$

where $\mathrm{K}_{1,2}$ are the integration constants. The even Mathieu function with real characteristic value $a$ and parameter $q$ is denoted $C\left(a, q, \xi+\xi_{0}\right)$, the odd solution by $S\left(a, q, \xi+\xi_{0}\right)$ and their derivatives with respect to the $\xi+\xi_{0}$ variable (can be complex) are $\dot{C}\left(a, q, \xi+\xi_{0}\right)$ and 
$\dot{S}\left(a, q, \xi+\xi_{0}\right)$, respectively.

The solution of the Mathieu equation for given $a, q, \xi$, $\xi_{0}$, and initial conditions $\left(u_{i}, \dot{u}_{i}\right)$ can be presented as follows:

$$
\begin{aligned}
& u(\xi)=\frac{1}{P(a, q)}\left[\dot{u}_{i} Q\left(a, q, \xi, \xi_{0}\right)+u_{i} L\left(a, q, \xi, \xi_{0}\right)\right] \\
& \dot{u}(\xi)=\frac{1}{P(a, q)}\left[\dot{u}_{i} \dot{Q}\left(a, q, \xi, \xi_{0}\right)+u_{i} \dot{L}\left(a, q, \xi, \xi_{0}\right)\right]
\end{aligned}
$$

where

$$
\begin{aligned}
& P(a, q)=\dot{C}\left(a, q, \xi_{0}\right) S\left(a, q, \xi_{0}\right)-C\left(a, q, \xi_{0}\right) \dot{S}\left(a, q, \xi_{0}\right) \\
& Q\left(a, q, \xi, \xi_{0}\right)= C\left(a, q, \xi+\xi_{0}\right) S\left(a, q, \xi_{0}\right) \\
&-C\left(a, q, \xi_{0}\right) S\left(a, q, \xi+\xi_{0}\right) \\
& L\left(a, q, \xi, \xi_{0}\right)= \dot{C}\left(a, q, \xi_{0}\right) S\left(a, q, \xi+\xi_{0}\right) \\
&-C\left(a, q, \xi+\xi_{0}\right) \dot{S}\left(a, q, \xi_{0}\right)
\end{aligned}
$$

and

$$
\begin{gathered}
\left\{\begin{array}{l}
K_{1}=\frac{1}{P(a, q)}\left(-\dot{u}_{i} C\left(a, q, \xi_{0}\right)+u_{i} \dot{C}\left(a, q, \xi_{0}\right)\right) \\
K_{2}=\frac{1}{P(a, q)}\left(\dot{u}_{i} S\left(a, q, \xi_{0}\right)-u_{i} \dot{S}\left(a, q, \xi_{0}\right)\right)
\end{array}\right. \\
\text { or }\left\{\begin{array}{l}
K_{1}=\frac{\dot{u}_{i}}{\dot{S}(a, q, 0)} \text { for } \xi_{0}=0 . \\
K_{2}=\frac{u_{i}}{C(a, q, 0)}
\end{array}\right.
\end{gathered}
$$

Eq 2 is essentially the same as eq 3 and both are equally useful. However, eq 3 has some advantages in association with the matrix method.

It should be recognized that the analytical method of solution of eq 1 does not require anything else to be complete. However, for evaluation of the analytical method it is beneficial (but not necessary) to follow the matrix method formalism (see, for example $[5,6]$ ), which is based on a pair of independent solutions for initial conditions $\left(u_{i}, \dot{u}_{i}\right)=(1,0)$ :

$$
\begin{aligned}
& u(\xi)=\frac{L\left(a, q, \xi, \xi_{0}\right)}{P(a, q)}=m_{11} ; \dot{u}(\xi)=\frac{\dot{L}\left(a, q, \xi, \xi_{0}\right)}{P(a, q)}=m_{21}, \\
& \text { and for }\left(u_{i}, \dot{u}_{i}\right)=(0,1): \\
& u(\xi)=\frac{Q\left(a, q, \xi, \xi_{0}\right)}{P(a, q)}=m_{12} ; \dot{u}(\xi)=\frac{\dot{Q}\left(a, q, \xi, \xi_{0}\right)}{P(a, q)}=m_{22} .
\end{aligned}
$$

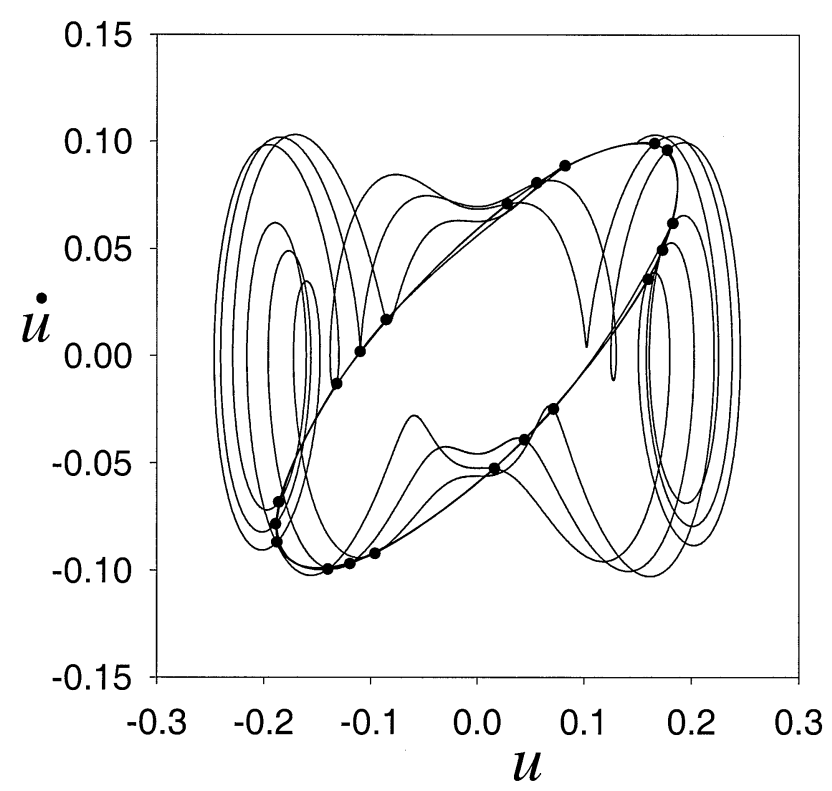

Figure 1. Ion trajectory calculated according to eqs 3,5 is presented for $\left(u_{i}, \dot{u}_{i}\right)=(0.15,0.1)$ and $a=0, q=0.4$ together with the ellipse $\xi=n \pi$ for $\xi_{0}=1 / 5 \pi$.

For any vector of initial conditions $\left(u_{i}, \dot{u}_{i}\right)$ we have:

$$
\begin{aligned}
& \left(u_{i}, \dot{u}_{i}\right)=M \cdot\left(u_{i}, \dot{u}_{i}\right)=\left(\begin{array}{ll}
m_{11} & m_{12} \\
m_{21} & m_{22}
\end{array}\right) \cdot\left(u_{i}, \dot{u}_{i}\right)= \\
& \left(\begin{array}{c}
\frac{1}{P(a, q)}\left[\dot{u}_{i} Q\left(a, q, \xi, \xi_{0}\right)+u_{i} L\left(a, q, \xi, \xi_{0}\right)\right], \\
\frac{1}{P(a, q)}\left[\dot{u}_{i} \dot{Q}\left(a, q, \xi, \xi_{0}\right)+u_{i} \dot{L}\left(a, q, \xi, \xi_{0}\right)\right]
\end{array}\right)
\end{aligned}
$$

If one limits the time variable $\xi$ to $n \pi$ values ( $n$ complete RF periods) and assumes that $\xi_{0}$ is zero, then matrix M is equal to the transfer matrix. The matrix method requires a numerical method to calculate an ion trajectory over one cycle if $\xi_{0} \neq 0$. These limitations are not required in the analytical approach and eqs 4 and 5 are sufficient for any $\xi$ and $\xi_{0}$. In Figure 1 the solution (see eq 3 ) is presented for $\left(u_{i}, \dot{u}_{i}\right)=(0.15,0.1)$ and $a=0, q=$ 0.4 together with the ellipse $n \pi$ for $\xi_{0}=1 / 5 \pi$. Intersections between the ellipse and trajectory happen every $\xi=n \pi$, as should be.

\section{Stability Parameterization}

According to the Liouville theorem:

$$
\frac{\dot{C}\left(a, q, \xi+\xi_{0}\right) S\left(a, q, \xi+\xi_{0}\right)-C\left(a, q, \xi+\xi_{0}\right) \dot{S}\left(a, q, \xi+\xi_{0}\right)}{\dot{C}\left(a, q, \xi_{0}\right) S\left(a, q, \xi_{0}\right)-C\left(a, q, \xi_{0}\right) \dot{S}\left(a, q, \xi_{0}\right)}=1
$$

This can be true only if the nominator and denominator are independent of the time variable $\xi$ and initial phase 


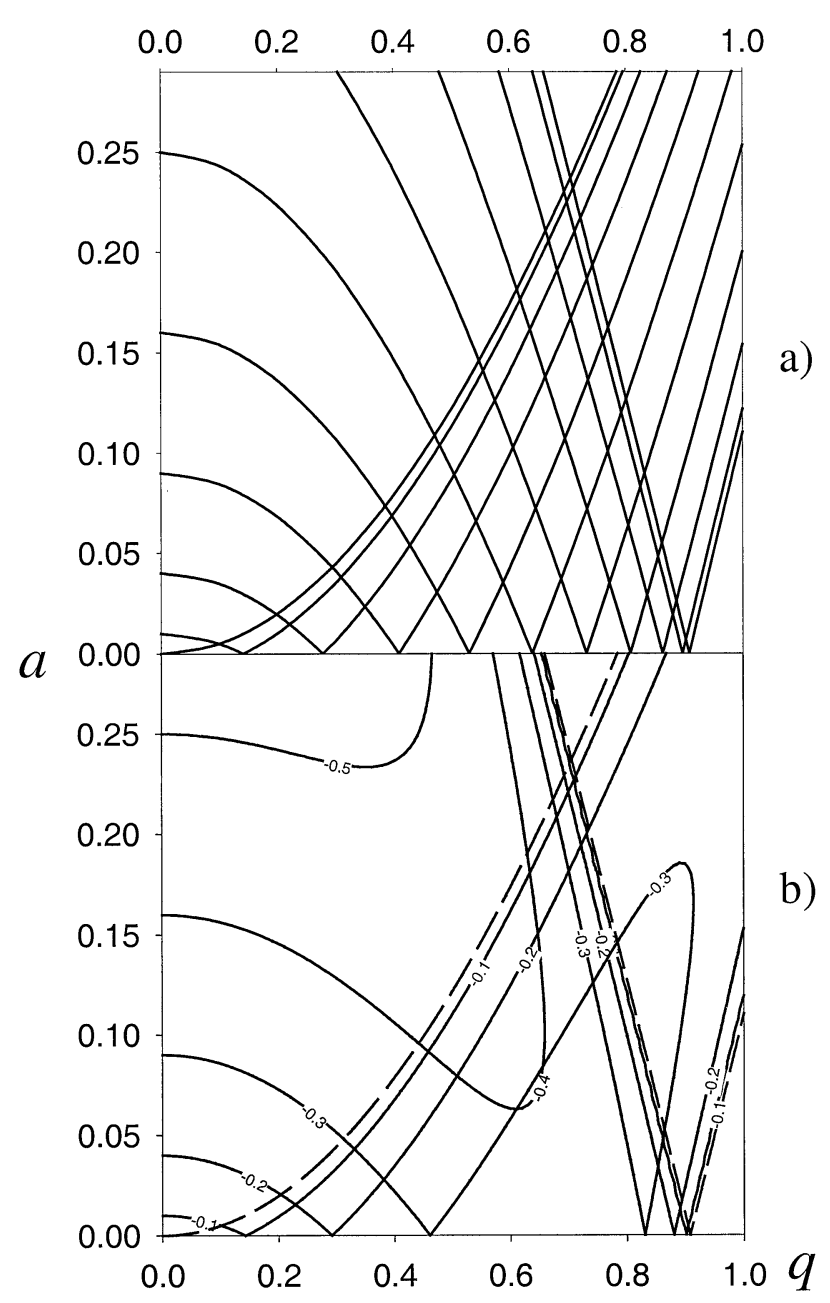

Figure 2. The first region of stability. (a) Graphing of iso- $\beta$ lines was conducted according to eq 8. (b) Schematic presentation of $P(a, q)$ (according to eq 7) as a contour plot. Only real values corresponding to stable solution are presented. The algorithm for drawing the contour lines is not precise enough. Therefore, $P(a, q)$ $=0$ dashed lines are used to border the stability region.

$\xi_{0}:$

$$
\begin{aligned}
\dot{C}\left(a, q, \xi+\xi_{0}\right) S\left(a, q, \xi+\xi_{0}\right) & -C\left(a, q, \xi+\xi_{0}\right) \dot{S}\left(a, q, \xi+\xi_{0}\right) \\
& \quad \dot{C}\left(a, q, \xi_{0}\right) S\left(a, q, \xi_{0}\right)-C\left(a, q, \xi_{0}\right) \dot{S}\left(a, q, \xi_{0}\right) \\
= & \dot{C}(a, q, 0) S(a, q, 0)-C(a, q, 0) \dot{S}(a, q, 0) \\
= & -C(a, q, 0) \dot{S}(a, q, 0) \\
= & P(a, q)
\end{aligned}
$$

$P(a, q)$ is a real number for a stable trajectory and a complex number for an unstable one. As can be seen in Figure 2, it is fully sufficient to establish the stability boundaries. To understand its advantage, comparison with the transfer matrix is again useful and it leads us to the stability requirement $\left|m_{11}+\mathrm{m}_{22}\right| \leq 2$ or, introducing a stability number $\beta$ (Mathieu characteristic exponent) for $n=1$ :

$$
\begin{aligned}
& m_{11}+m_{22}=2 \cos (\pi \beta)=\frac{1}{P(a, q)} \\
& \times\left(\begin{array}{c}
C(a, q, \pi) \dot{S}(a, q, 0)-\dot{C}(a, q, 0) S(a, q, \pi) \\
+C(a, q, 0) \dot{S}(a, q, \pi)-\dot{C}(a, q, \pi) S(a, q, 0)
\end{array}\right) \\
& \beta= \pm \frac{1}{\pi} \arccos \left(\frac{1}{2}\left(m_{11}+m_{22}\right)\right) \\
& =\frac{1}{\pi} \arccos \left(\frac{C(a, q, \pi)}{C(a, q, 0)}\right) \\
& =\frac{1}{\pi} \arccos \left(\frac{\dot{S}(a, q, \pi)}{\dot{S}(a, q, 0)}\right)
\end{aligned}
$$

Although all functions in $\mathrm{M}$ formally depend on the initial phase $\xi_{0}$ and the time variable $\xi$, the matrix method defines $\xi_{0}=0$ and $\xi=\pi$. The stability number $\beta$ is defined for $\xi_{0}=0$ and $\xi=\pi$ and is complex for unstable trajectories. $P(a, q)$ is independent of the time variable and does not require such restrictive definitions. Obviously, $\beta$ and $P(a, q)$ are mutually interdependent and allow definition of the stability boundaries. In Figure 2 the first region of stability is presented. Two methods of its calculation were used: Explicit graphing of iso- $\beta$ lines (according to eq 8 , which by itself is much easier than the traditional algorithm, for example $[5,6])$ and schematic presentation of $P(a, q)$ (according to eq 7 ). It can be seen that besides the stability of the ion trajectory, the amplitude $\left|P(a, q)^{-1}\right|$ also reflects the trajectory size and approaches infinity on the stability boundary $[P(a, q)=0$, dashed lines]. Position $(a, q)$ of the extreme points of the stability regions is easily determined by solution of the system of eq $P(a, q)=0$ for all directions. In addition, $P(a, q)$ does not have singularities or uncertainties similar to $\beta$.

\section{Investigation of the Quadrupole Field Acceptance}

In the particular case of the quadrupole theory $[7,8]$, $\xi=\frac{\omega t}{2}(t=$ time, $\xi=$ dimensionless time variable, $\omega=$ the main trapping RF angular frequency) and $\xi_{0}$ is the initial phase of the main trapping frequency. The period of the main trapping frequency is equal to $\pi$ in dimensionless units. The acceptance of an ideal quadrupole field with field radius $r_{0}$ is a function of $a, q,\left(u_{i}, \dot{u}_{i}\right), \xi$, and $\xi_{0}$. Ion beam transmission can be defined as the normalized integral of acceptance over the ion beam number density and velocity distributions [over $\left(u_{i}, \dot{u}_{i}\right)$ ] for given $a, q, \xi$, and $\xi_{0}$. Additional integration (averaging) over time and initial phase is also possible because the ion beam is assumed to be continuous and the initial distribution of the ion density is time independent. The 
ion density distributions (radial as well as axial) will not be considered in this work or, equally, can be assumed to be uniform. The influence of the velocity distributions (again, radial as well as axial) will be considered in subsequent papers. Because quadrupoles are normally operated under fixed stability conditions ( $a$ and $q$ ), the "ion guide" $(a=0)$ and "mass resolving" modes of operation ( $a$ and $q$ close to the apex of stability) are used as examples.

More advanced comparison of the explicit solution of the Mathieu equation with the matrix method allows the determination of $\mathrm{A}, \mathrm{B}$, and $\Gamma$ elements of the $n$th power of the transfer matrix $\mathrm{M}$ (for $\xi=\pi$, for definition of the transfer matrix elements see eq 3 and $[5,6])$ :

$$
\begin{aligned}
& A=\frac{m_{11}-m_{22}}{\sqrt{4-\left(m_{11}+m_{22}\right.}}, \\
& B=\frac{2 m_{12}}{\sqrt{4-\left(m_{11}+m_{22}\right)^{2}}}, \\
& \Gamma=\frac{-2 m_{21}}{4-\left(m_{11}+m_{22}\right)^{2}} ; \text { and } B \Gamma-A^{2}=1 .
\end{aligned}
$$

Let us consider $u_{\max }$ as a parameter for estimation of the maximum displacement of the ion trajectory in the quadrupole field. $u_{\max }=\sqrt{B \varepsilon}$ is defined for $n=1$ (one full RF cycle) and can be considered as a function of $a$, $q,\left(u_{i}, \dot{u}_{i}\right)$ and $\varepsilon_{0}$. An analogous expression can be obtained for the maximum radial speed, $\dot{u}_{\max }=\sqrt{\Gamma \xi}$, where $\varepsilon=\Gamma u_{i}^{2}+2 A u_{i} \dot{u}_{i}+B \dot{u}_{i}^{2}$.

For given $\xi_{0}$, the $u_{\text {max }}$ parameter does not necessarily represent the absolute maximum radial displacement of the ion trajectory with $\left(u_{i}, \dot{u}_{i}\right)$ for given $a$ and $q$. However, its plot over $\xi_{0} \in[0, \pi]$ reveals the portion of rejected initial conditions without having to calculate trajectories explicitly. The " $u_{\max }$ versus $\xi_{0}$ " diagrams represent the acceptance of the ideal quadrupole field for given $a, q,\left(u_{i}, \dot{u}_{i}\right)$ as a function of $\xi_{0}$ and are presented in Figures 3 and 4. For $a=0, q=\{0.2,0.4,0.6$, $0.7,0.8,0.85\}$, and $\left(u_{i}, \dot{u}_{i}\right)=(0.15,0.1)$ (see Figure 3$)$ the acceptance is wide. Notice that for $q=0.2$ the RF field strength is not enough to securely confine ions with given $\left(u_{i}, \dot{u}_{i}\right)$. The circular shape of $u_{\max }$ versus $\xi_{0}$ is characteristic of the adiabatic approximation (or adiabaticity). While nearly stagnant for $q<0.7$, acceptance decreases rapidly as the stability boundary is approached $q>0.7$. $\dot{u}_{\max }$ increases steadily with increase of the $q$ parameter which leads to an increase of the RF field contribution to the total ion energy. For $a=\{0.2$, $0.23,0.2369\}, q=0.705996$, and $\left(u_{i}, \dot{u}_{i}=(0.15,0.1)\right.$ (see Figure 4$), u_{\max }$ often exceeds $r_{0}$ and the RF field contribution to the total ion energy increases exponentially.

Also, the acceptance can be expressed as the integral of the following step function:

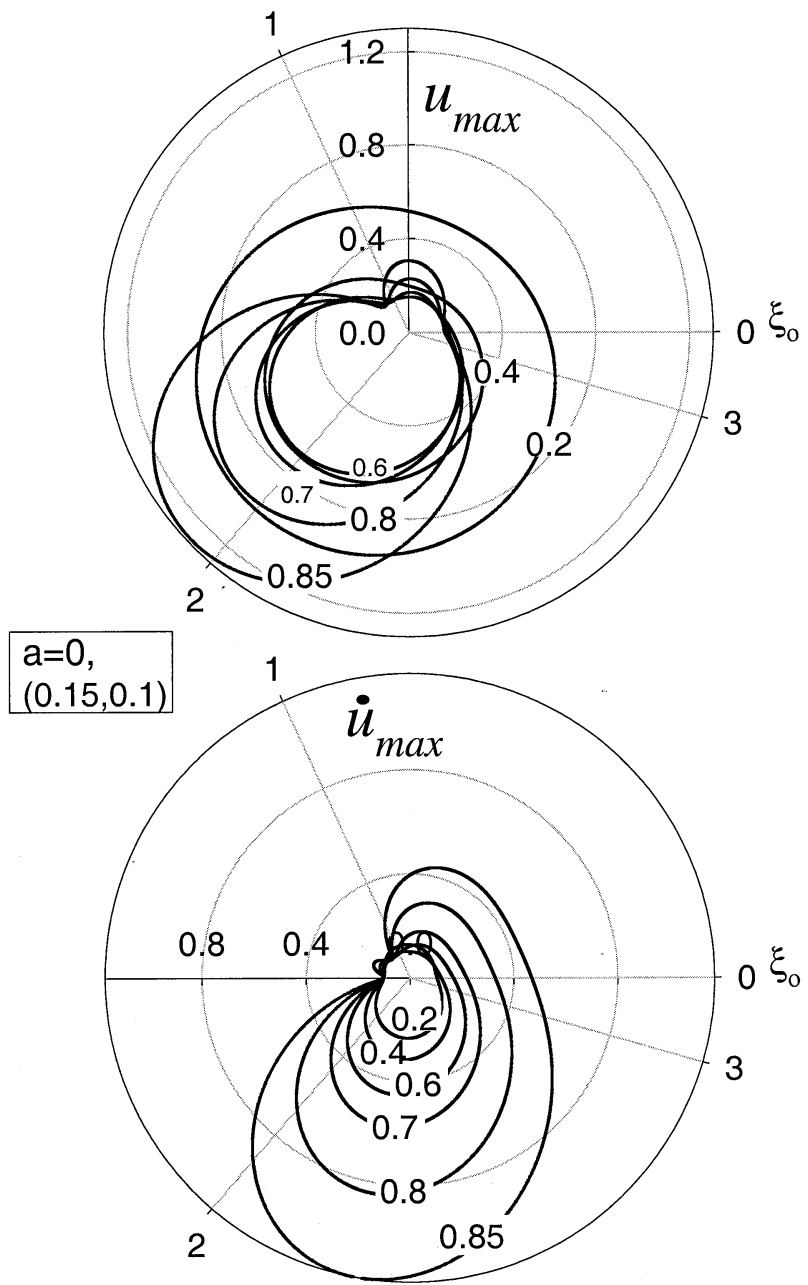

Figure 3. The $u_{\max }$ and $\dot{u}_{\max }$ versus $\xi_{0}$ diagrams for $a=0, q=$ $\{0.2,0.4,0.6,0.7,0.8,0.85\}$, and $\left(u_{i}, \dot{u}_{i}\right)=(0.15,0.1)$.

$$
\begin{aligned}
& \operatorname{ac}\left(a, q, \xi_{0},\left(u_{i}, \dot{u}_{i}\right)\right)=\left\{\begin{array}{l}
1, \text { if } u_{\max }<1 \\
0, \text { if } u_{\max } \geq 1
\end{array}\right. \\
& \operatorname{Ac}\left(a, q,\left(u_{i}, \dot{u}_{i}\right)\right)=\frac{1}{\pi} \int^{\pi} a c\left(a, q, \xi_{0},\left(u_{i}, \dot{u}_{i}\right)\right) d \xi_{0} .
\end{aligned}
$$

Acceptance (Ac) as a function of $\dot{u}_{i}$ is presented in Figure $5\left(u_{i}\right.$ is fixed at 0.15$)$. Looking at the acceptance of the ideal quadrupole field in the case of $a=0$ (see Figure 5a), two undesirable effects are evident: low confinement strength of the RF field in the low $q$ region and high RF driven radial displacement (outside of the quadrupole field radius) for high $q$ values. Optimum confinement appears to occur near $q=0.7$, however, other concerns must usually be taken into consideration such as: Stability of a desired mass range, the RF field contribution to the ion energy, size of the housing, and ion optics (higher $q$ leads to a larger radial displacement) and simplicity of experimental realization. For 

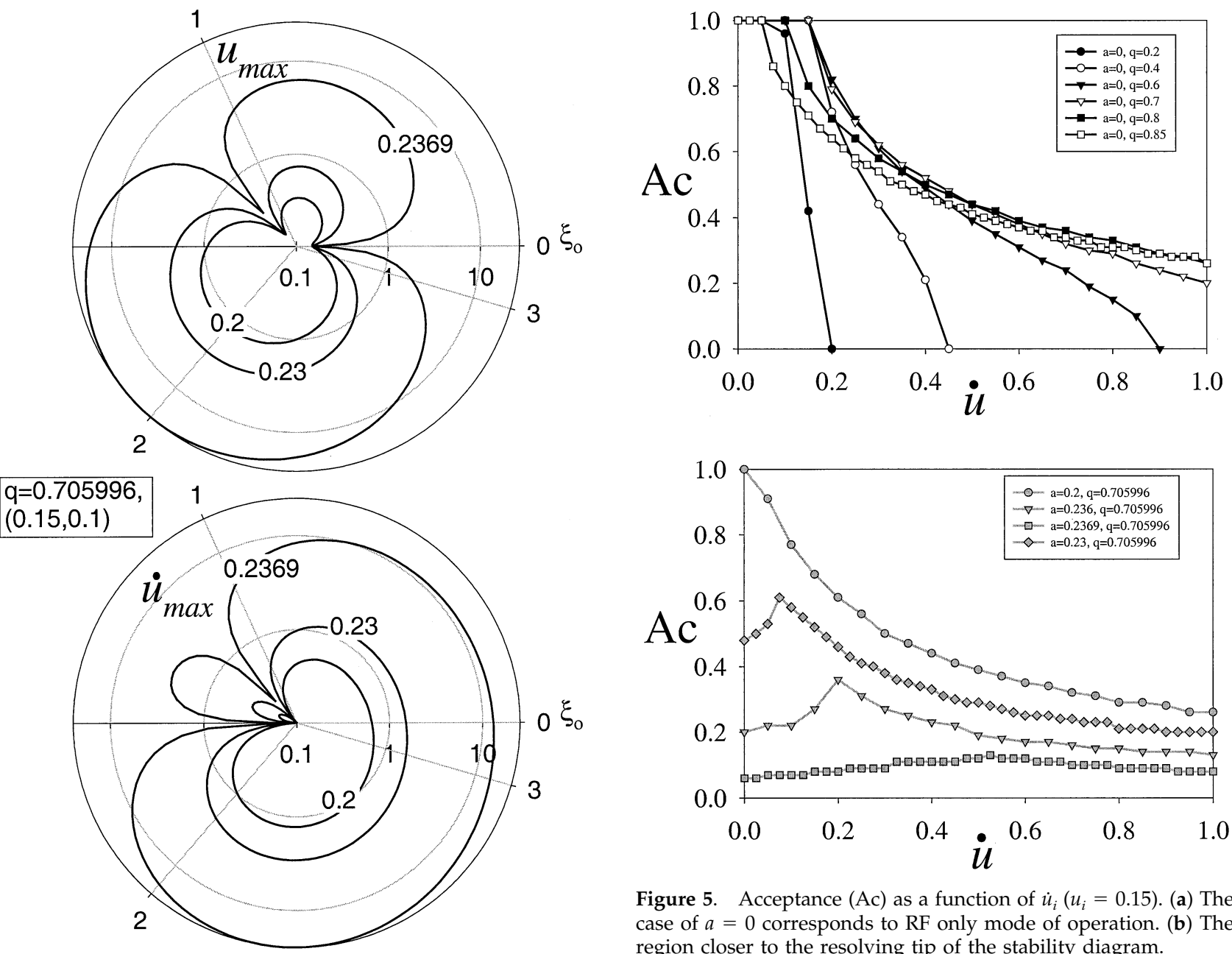

Figure 5. Acceptance (Ac) as a function of $\dot{u}_{i}\left(u_{i}=0.15\right)$. (a) The case of $a=0$ corresponds to RF only mode of operation. (b) The region closer to the resolving tip of the stability diagram.

Figure 4. The $u_{\max }$ and $\dot{u}_{\max }$ versus $\xi_{0}$ diagrams for $a=\{0.2,0.23$, $0.2369\}, q=0.705996$, and $\left(u_{i}, \dot{u}_{i}\right)=(0.15,0.1)$. Notice the exponential scale for $u_{\max }$ and $\dot{u}_{\max }$.

the region closer to the resolving tip of the stability diagram (first region, see Figure $5 b$ ), a clear maximum of the acceptance function can be observed and the overall acceptance is reduced dramatically.

Acceptance as a function of $u_{i}$ is presented in Figure $6\left(\dot{u}_{i}\right.$ is fixed for each figure) and does not involve the matrix method. Contrary to the initial ion velocity, the initial position distribution has an obvious limitation: The RF field radius $r_{0}, u_{i}$ for a transmitted ion trajectory is defined in the interval from $-r_{0}$ to $r_{0}$ (or from -1 to 1). In this case, considering the acceptance, it is sufficient to generate a contour plot, which represents the time evolution of the $u(\xi)$ coordinate integrated over $\xi_{0}$ starting from all possible $u_{i}$ values in the interval $(-1$, $1)$. For the contour plots presented here, $\xi$ was varied from 0 to $5 \pi$. Contours for $u(\xi)$ also were limited to the interval $(-1,1)$. As one can see in the plot for $\dot{u}_{i}=0.1$, $a=0.2369, q=0.705996$, only ions with initial $u_{i}$ between the two marker lines will be accepted. For $\dot{u}_{i}=$ $0.15, a=0.2369, q=0.705996$, acceptance is virtually zero. For the $a=0$ mode of operation, acceptance is broad allowing a wide range of $u_{i}$ to be accepted.

The analytical method offers more flexibility in the investigation of the acceptance without involvement of the matrix or numerical methods. For example, averaging over the initial phase $\xi_{0}$ is straightforward as is application of any ion number density or velocity distribution functions. Acceptance is a multidimensional function and its estimation and presentation is a complex problem, although in the case of the analytical approach, limited only by our imagination. Examples employed in this paper were selected in order to demonstrate how the analytical method (in conjunction with algebraic method to compute Mathieu functions) could be used to calculate acceptance, and by no means represent a comprehensive study of this matter.

\section{Frequency Spectrum of a Stable Ion Trajectory Resonance}

According to Floquet's theorem, any Mathieu function can be written in the form $f(\xi) e^{-i \beta \xi}$. This form can be presented as: $f(n \omega t) e^{-i \beta \frac{\omega t}{2}}$ (recall that $\xi=\frac{\omega t}{2}$ ) and its Fourier transform is equal to: 


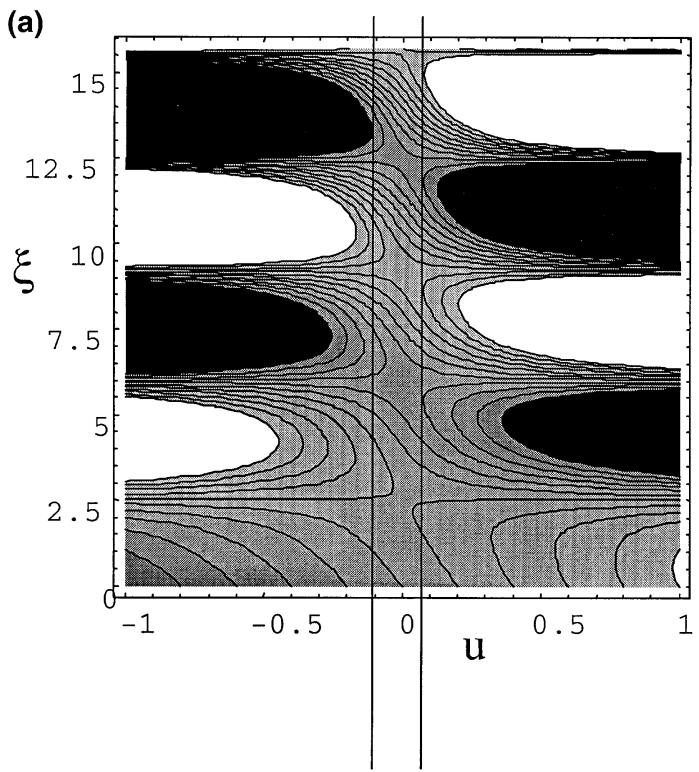

$$
\begin{aligned}
F\left(\omega_{i}\right)= & K_{3}\left(\delta\left(-2 \omega_{i}+2 n \omega+\beta \omega\right)\right. \\
& \left.-\delta\left(2 \omega_{i}+2 n \omega-\beta \omega\right)\right),
\end{aligned}
$$

for which the coefficients are different from zero only for $\omega_{i}=\omega_{n}$, where

$$
\frac{\omega_{n}}{\omega}=\left|n+\frac{\beta}{2}\right|, n=0, \pm 1, \pm 2, \ldots
$$

Here $\delta(\mathrm{x})$ represents the Dirac delta function and $\mathrm{K}_{3}$ is a scaling constant.

A trajectory in an ideal quadrupole field (see eq 3), averaged over initial phase, can be expressed in the following manner:

$$
\begin{aligned}
& \frac{u(\xi)}{u_{i}}= \\
& \frac{1}{\pi P(a, q)} \int_{0}^{\pi}\left(\alpha Q\left(a, q, \xi, \xi_{0}\right)+L\left(a, q, \xi, \xi_{0}\right)\right) d \xi_{0}, \\
& \alpha=\frac{\dot{u}_{i}}{u_{i}} .
\end{aligned}
$$

In this form, normalized on the initial coordinates, the trajectory starts from $\frac{u(0)}{u_{i}}=1$ and progresses as a function of $\xi$. Characteristics of this trajectory are defined by the parameters $a, q$, and its displacement by $\alpha$. The trajectory is quasiperiodic and its power spectrum includes multiple harmonics $\omega_{n}$.

For $a=0.2369$ and $q=0.705996$ the stability number

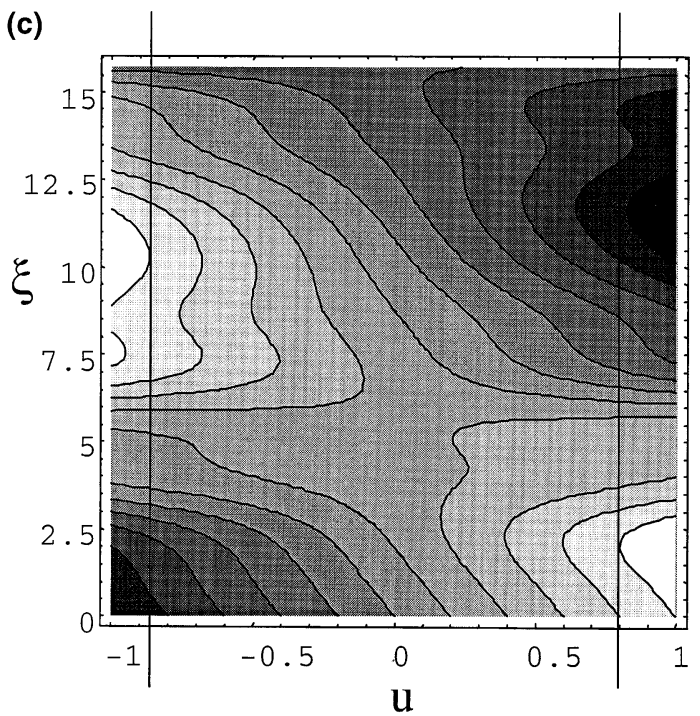
$\beta$ is equal to 0.993058 (calculated using eq 8). Using $\alpha=$ $\{0.5,2\}$, the power spectrum of $\frac{u(\xi)}{u_{i}}$ trajectory can be calculated and is presented in Figure 7. Resonances appear at $\frac{\omega_{0}}{\omega}=0.496529 \approx \frac{\omega_{-1}}{\omega}$ and $\frac{\omega_{1}}{\omega}=1.49653$ $\approx \frac{\omega_{-2}}{\omega}$, which corresponds to eq 12. Parameter $\alpha$ does not change the position of resonances, affecting only the size of the trajectory. The width of resonances is predetermined by the residence time of an ion in the quadrupole field. For $a=0$ and $q=0.4$ the stability number $\beta$ is equal to 0.292566 and resonances are observed at $n=0, \pm 1$, and \pm 2 .

\section{Conclusions}

Traditionally, calculations of the stability of ions' motion and their acceptance in an ideal quadrupole field with periodic time varying potentials have used the matrix method. Recent developments that have resulted in obtaining closed formulae of the Mathieu functions

Figure 6. Acceptance as a function of $u_{i}\left(\dot{u}_{i}\right.$ is fixed for each figure). $u_{i}$ is defined in the interval $(-1,1)$; the time variable $\xi$ was varied from 0 to $5 \pi$. The range of the contour plot for $u(\xi)$ is limited to $(-1,1)$. (a) $\dot{u}_{i}=0.1, a=0.2369, q=0.705996$; (b) $\dot{u}_{i}=$ $0.15, a=0.2369, q=0.705996$; (c) $\dot{u}_{i}=0.15, a=0, q=0.4$. 

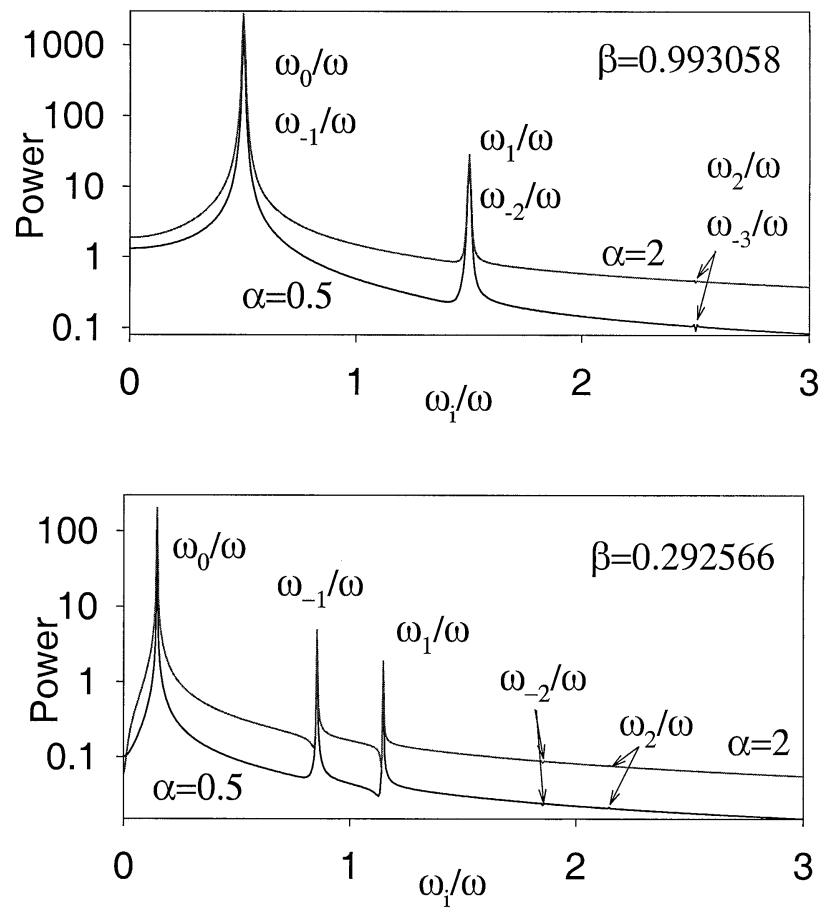

Figure 7. Power spectrum of the ion trajectory in the ideal quadrupole field. (a) $a=0.2369$ and $q=0.705996 ;$ (b) $a=0$ and $q=0.4$.

allow a fast and efficient means for analytical expression and calculation of parameters of the stability diagrams for a quadrupole mass filter and for a quadrupole ion trap. This method is also applicable to the higher regions of stability without limitations. It is still limited to cos trapping waveforms and cannot be used directly for solution of the more general Hill equation. This work involves heavily the matrix method for comparative study only. The analytical method allows the analytical formulation (and calculation) of an ion trajectory over unlimited number of cycles and does not need the matrix method. It also offers simple expressions for the stability conditions, acceptances, and resonances. Subsequent publications will investigate the energy of the ions in the presence of the RF field in different stability regions. Also, the influence of collisions with buffer/target gas on stability, acceptance, transmission, and spatial focusing/distribution of the ions will be considered.

\section{Acknowledgments}

The author would like to thank Dr. Scott Tanner and Dr. Dmitry Bandura for their helpful discussions, suggestions, and support.

\section{References}

1. Frenkel, D.; Portugal, R. Algebraic Methods to Compute Mathieu Functions. J. Phys. A Math. Gen. 2001, 34, 3541-3551.

2. Kokkorakis, G. C.; Roumeliotis, J. A. Power Series Expansions for Mathieu Functions with Small Arguments. Math. Comput. 2001, 70, 1221-1235.

3. O'Dell, D. H. J. Dynamical Diffraction in Sinusoidal Potentials: Uniform Approximations for Mathieu Functions. J. Phys. A Math. Gen. 2001, 34, 3897-3925.

4. htpp://functions.wolfram.com. 2003.

5. Sudakov, M.; Konenkov, N.; Douglas, D. J.; Glebova, T. Excitation Frequencies of Ions Confined in a Quadrupole Field with Quadrupole Excitation. J. Am. Soc. Mass Spectrom. 2000, 11, $10-18$.

6. Konenkov, N. V.; Sudakov, M.; Douglas, D. J. Matrix Methods for the Calculation of Stability Diagrams in Quadrupole Mass Spectrometry. J. Am. Soc. Mass Spectrom. 2002, 13, 597-613.

7. Dawson, P. H. Quadrupole Mass Spectrometry and Its Applications. American Institute of Physics: Woodbury, 1995.

8. March, R. E.; Hughes, R. J. Quadrupole Storage Mass Spectrometry. John Wiley and Sons: New York, 1989. 